$\boldsymbol{A}$

\title{
CHEMICAL ACCOUNT
}

or

\section{VARIOUS DROPSICAL FLUIDS;}

WirH

REMARKS ON THE NATURE OF THE ALKALINE MATTER CONTANED IN THESE FLUIDS, AND ON THE SERUM OF THE BLOOD.

By ALEXANDER MARCET, M.D. F.R.S. ONE OF THE PBYSICIANS TO GUY'S HOBPITAL.

Read June 18, 1811.

THE attention of the Society having been lately directed by an interesting communication of $\mathrm{Mr}$. Astley Cooper, to the singular species of dropsy called Spina Bifida; and various opportunities having been afforded me by that able and indefatigable inquirer, of examining the fluid which the spinal canal secretes in this disease, I made an analysis of that fluid, with a view to submit it to the Society.

It occurred to me in the course of this inquiry, 
that although there existed some detached accounts of most of the other species of dropsical fluids, yet a careful re-examination of these by the same individual, and by similar methods, would not be without interest. With this view, after analysing the fluid of Spina Bifida, I examined in succession those of hydrocephalus, and of dropsy of the pericardium, chest, and abdomen*. I afterwards inquired into some facts, respecting the blood itself, which seemed to require revision, and to which my attention had been naturally directed by the preceding researches.

This inquiry has thus become more extensive than was at first intended, and from the accidental circumstance from which it originated, the order of the subject has been in a manner inverted, since the serum of the blood was amongst the last fluids examined, though it is the obvious source from which all animal fluids are derived.

This, however, is of no material consequence, and I have thought it preferable, both for the sake of stating the facts exactly as they occurred, and also for my own convenience, to preserve in my narrative the same order which prevailed in the series of experiments.

* The examination of the fluid of hydrocele, of hydatids, and that from a tumor of the thyroid gland, was afterwards added to this inquiry. 


\section{I. Of the Fluid of SpINA Bifida.}

A. General properties of the ftuid.-In several specimens of this fluid which I examined at different periods, the following properties were observed:

1. Its specific gravity was $1007^{*}$, water being 1000.

2. It was perfectly pellucid and free from smell, when quite recent; and it continued so for some days after it had been collected.

3. It was alkaline, as appeared from its imparting a green colour to paper stained with violets.

4. Muriatic acid produced no appearance of coagulation on being added to it. Sulphuric acid occasioned a slight turbidness, particularly when heat was applied. Nitric acid produced a white cloud, which immediately subsided, but disappeared by agitation.

5. Oxymuriat of mercury produced no effect at first, but on applying heat, a slight opacity was perceived.

6. Infusion of galls produced a slight turbidness, but no distinet precipitation.

* In one instance it was found as low as 1006,6. 
7. Alcohol occasioned no change whatever.

8. Oxalat of lime produced but a very slight cloudiness.

9. Both acetat of lead and nitrat of silver formed abundant precipitates.

10. Muriat of barytes produced no perceptible effect on the fluid in its unconcentrated state.

11. Solutions of oxymuriat of platina did not occasion any precipitate.

12. On applying a boiling heat to the fluid, neither coagulation, nor any precipitation took place. But on concentrating it by evaporation, a white greasy pellicle appeared, which became at last quite dry and brittle, emitting during the process a faint animal smell, not unlike that of saliva when heated in a similar manner. On pushing the heat still farther, there remained a half charred, spungy, brown, animal substance, covering or mixed with a saline mass confusedly crystallized.

13. Water being poured upon this mass, the greater part of the animal matter remained undissolved. Yet a portion of it was dissolved, together with the saline matter, as was obvious from the brownish colour of the filtered clear fluid, and the pellicle which re-appeared on a second or even third repetition of this process. 
B. Nature and proportion of the animal matter.-Upon considering the results just related, especially those marked $4,5,6$, and 13 , and comparing them with the observations published at different periods by Dr. Bostock and other chemists*, respecting the detection and discrimination of the animal fluids, it will appear that the animal matter contained in this fluid consists almost entirely of a soluble uncoagulable substance, which may be called extractive or muco-extractive matter, with a mere vestige of albumen, and without any sensible quantity of gelatine. The slight cloudiness produced by the infusion of galls in Exp. 6. cannot, I think, be considered as indicative of the presence of jelly; on the contrary, from the dense precipitate which tannin forms with the smallest quantities of gelatine, and the turbidness which it is known to occasion in solutions containing albumen, there seems to be every reason to ascribe the effect in question to

* See in particular Dr. Bostock's essays, in Nicholson's Journal, vol. xi. page 244, and vol. xiv. page 140; and in the MedicoChirurgical Transactions, vol. i. page 147.-See also Mr. Brande's paper in the Philos. Transact. for 1809, page 373; and Dr. Pearson's papers on expectorated matter and pus, in the Philos. Transact. for 1809 and 1810.

Dr. Bostock, in the second of the éssays iust quoted, gave a summary account of his examination of the fluid of Spina Bifida, which, in a great degree, will be found to correspond with this. But the differences which may be observed are in a great measure accounted for, by our improved knowledge of the mode of conducting these researches, an improvement to which Dr. Bostock has himself largely contribuled. 
the small quantity of albumen known to exist in the. fluid.

As to the proportion of the animal matter, I tried to ascertain it by the following method :

1. 600 grs. of the fluid having been evaporated to dryness in a platina crucible, the residue, when Brought by a sand heat to the spungy, brittle, halfcharred state before described, weighed $6,5 \mathrm{grs}$.

2. The mass being then made red hot, so as to buin entirely the animal matter (which was thus. reduced to a few particles of ash or charcoal), its weight was diminished to 5,2 grs. from which $I$ inferred that the weight of the animal matter contained in 600 grs. of the fluid, and desiccated in the mode I have just described, amounted to only 1,3 grs. * which is equivalent to $2,2 \mathrm{grs}$. in 1000 of the fluid.

C. Quantity of the saline matter.-After having burnt off the animal matter in the way just related $t$,

* A small allowance should, in strict accuracy, be made for the coaly matter, the quantity of which, however, would have been scarcely ponderable.

+ It may be objected to the method of incineration, which has been used for the separation of the salts from the animal matter in all these analyses, that the ammoniacal salts (if any be present in these fluids) would be driven off by that process. But as the co-existence of ammoniacal salts with a fixed alkali in excess is highly improbable, and as the fluids in question give out no ammonia 
the quantity of the remaining saline mass, in three successive experiments, made with different speci. mens of the fluid, was found to be as follows :

Exp. 1. 400 grs. of the fluid yielded 3,5 grs:

2. $200 \quad-\quad-\quad-\quad-1,7$

3. $300 \quad-\quad-2,9$

So that $900 \mathrm{grs}$. of the fluid yielded 8,1 grs. of saline matter.

The average quantity of saline matter, therefore, when dried at an incipient red heat, appears to be 9 grs. * in 1000 of the fluid.

D. Nature and proportions of the saline ingre dients.-This part of the subject was investigated in the following manner:

1. Water being added to $5 \mathrm{grs}$. of the saline mass, the whole was readily dissolved, with the exception of a few white light flakes, which remained floating in the fluid, and appeared to be vestiges of animal matter. This solution, on being

ammonia on adding to thern caustic potash, there is every reason to suppose that the ammonia which is, under certain circumstances, yielded by these fluids, exists in them in a state of intimate union with the animal matter, or is actually formed in consequence of the processes of decomposition to which all kinds of animal matter are liable.

* With the addition of the minute quantity of insoluble salts mixed with the coaly matter. 
filtered and slowly evaporated to dryness, was resolved into clusters of cubic crystals of muriat of soda, surrounded by a scanty pellicle of saline matter not crystallized, and evidently deliquescent.

2. This saline mass effervesced with acids, and being re-dissolved in water, had the following properties :

a. It changed to a green colour paper stained with violets.

b. It formed copious precipitates with nitrat of silver, and distinct, though inconsiderable ones, with nitrat and muriat of barytes; independently of the uncombined alkali, which had been previously saturated by acetic acid.

c. Oxalat of ammonia, prussiat of potash, caustic alkalis, and oxymuriat of platina, produced no precipitates whatever.

This solution, therefore, appeared to consist of muriat of soda, carbonat of soda, and a vestige of an alkaline sulphat.

3. The insoluble ash, or carbonaceous matter, which amounted but to a small fraction of a grain, being dissolved in a drop of diluted muriatic acid, was found to contain distinct traces of lime and 
iron, but no vestige of sulphuric acid. Phosphoric acid was also discovered in this solution (after the separation of the lime) by solutions of supercarbonat of ammonia, and of any magnesian salt.

4. In order to ascertain the proportions of the two principal ingredients of the fluid (muriat of soda, and soda), I had recourse to the following methods :

To a known quantity of the dry saline massacetic acid was added, with a view to combine with the soda, and to form an acetat which is soluble in alcohol. The mass being then again dried, and afterwards treated with alcohol, in order to dissolve the acetat of soda, and thus separate it from the muriat ; and the alcoholic solution being decanted off and evaporated to dryness, a residue, supposed to consist of acetat of soda, was obtained, which weighed between 17 and 18 per cent. of the mass *.

5. The next method which was tried consisted in precipitating the muriatic acid from a known

- This process having been performed upon very small quantities of saline matter, and no opportunity of repeating it having occurred, I relate these particulars rather with a view to state the method I used, than from any great confidence in the accuracy of the result. Another process apparently susceptible of much greater accuracy was employed in the analysis of serum, as will be seen bereafter. 
weight of the saline mass (previously acidulated by nitric acid), by nitrat of silver, and inferring from this precipitate the quantity of muriat of soda contained in the mass*. From this mode of operating it appeared that $100 \mathrm{grs}$. of the saline mass contained 85 grs. of muriat of soda, the remaining 15 parts consisting almost entirely of soda, brought to the state of carbonat or subcarbonat, as may be inferred from the effervescence with acids before noticed $t$.

This last result, which implies rather a larger quantity of soda from the former process (which yielded only between 17 and 18 parts of acetat of soda), I am inclined to consider as the most accurate, because it was obtained by a more simple and direct operation.

\section{E. Recapitulation. Upon the whole it may be}

* It has been ascertained by accurate experiments that 100 grs. of ignited muriat of soda yield 241 grs. of ignited muriat of silver. See Dr. Henry's paper in the Philos. Transact. for 1810.

+ I do not mean to assert, contrary to the opinion-generally. received, that the soda contained in the animal fluids does not exist there in a caustic state. But it is obvious that it must have been more or less carbonated during the process of combustion which the animal matter had undergone; and as a proof that the alkali originally exists in the caustic state, or in a peculiar state of combination with the animal matter, it may be observed, that if an acid be poured upon the dry residue, before the animal matter has been burnt off, no effervescence is observed. 
inferred from what precedes, that $1000 \mathrm{gra}$. of the fluid of spina bifida consist of

Water

- $\quad$

- 988,60 grs.

Muco-extractive animal matter, with

a vestige of albumen, brought to the state of a dry brownish spungy mass

just beginning to be charred * _. 2,20

Muriat of soda $t$ heated to incipient ignition - . - - $\quad$ - 7,65

Soda, brought to the state of subcarbonat and heated to incipient ignition, with a vestige of an alkaline sulphat - $\quad$ - $\quad$ - $\quad 1,35$

Phosphat of lime, and phosphat of iron, a quantity not exceeding $-\frac{0,20}{1000,00}$ grs.

which makes a total of $11,4 \mathrm{grs}$. of solid matter, heated in the manner just stated, in 1000 grs. of the fluid, 2,2 grs. of which consist of animal matter, and 9,2 grs. of saline ingredients.

* This standard of desiccation may appear somewhat indefinite. But it is extremely difficult to obtain perfect uniformity in analytical processes in which animal substances are concerned; and I beliere that, upon the whole, this mode of estimation will be found to yield more uniform results than those commonly employed.

+ I considered this salt, at the time this analysis was made, as the only muriat present. But from subsequent analyses of other animal fluids, I now think it probable, that in all of them, a minute portion of muriat of potash is to be found mixed with the muriat of soda, a circumatance which will be bereafter explained at full length. 
\$ II. Of the Fluid of Hyonocephalus Internus.

The experiments above related on the fluid of spina bifida were máde about 18 months ago. An opportunity having occurred in the course of last autumn, of examining the fluid which is effused in the ventricles of the brain in hydrocephalus, the result shewed, as might have been anticipated, that a close resemblance, if not a perfect identity; obtained between this fluid and that of spina bifida.

As the series of experiments in this analysis was conducted in the same manner as in the former, $I$ shall not trouble the Society with unnecessary chemical details, and shall confine myself to a general statement of results.

The specific gravity of this fluid was 1006,7; and the general effects of re-agents, the pellucidity of the fluid, and all its other obvious properties, corresponded exactly with those of the fluid of spina bifida.

Two different specimens of fluid of hydrocephalus, evaporated and dried as in the former analysis, exhibited similar results, but yielded a mean proportion of solid ingredients somewhat smaller than that obtained from the fluid of spina bifida.

The only new circumstance which occurred VOL. II. 
during this analysis, was the detection in one of the specimens examined, of a distinct trace of magnesia*, mixed with the coaly mass not soluble in water after the incineration of the residue; an appearance which might probably have also been remarked in the fluid of spina bifida, had this coaly residue been examined with the same degree of minutenesst.

Upon the whole, the solid contents of $1000 \mathrm{grs}$. of the fluid of hydrocephalus appear to consist of:

Water

Muco-extractive matter, with a ves-

tige of albumen, desiccated as in

the preceding analysis - $\quad-1,12$

Muriat of soda $\$$ - $\quad$ - $\quad$ - $\quad 6,64$

Subcarbonat of soda, with a vestige

of an alkaline sulphat - $\quad$ - 1,24

Phosphat of lime, with traces of phos-

phats of magnesia and of iron -

20

1000,00 grs.

which makes a total of $9,2 \mathrm{grs}$ of solid matter, consisting of 1,12 gr. of animal matter, and 8;08 of saline ingredients, in 1000 grs. of the fluid.

* I find thát Dr. Pearson, in his examination of expectorated matter, has been led to suispect the existence of magnèsia in that substance. See Ptilos. Transact. fot 1809, Part I. page 328.

t The whole of the saline contents of the coaly mass, when separated by inuriatio acid, and evaporated to dryness in a sand heat, amounted to about $0,1 \mathrm{gr}$. in each $500 \mathrm{grs}$. of the fluid.

¥ The note, page 350, respecting the probable existence of a small portion of thitriat of potash, applies equally to the present analysis. 
$\oint$ III. Inquiry into the Nature of the Auxaline MatTer contained in the Animal Fluids, exemplified in the Fluid of Hydrocephalus.

I selected for this inquiry a few grains of saline matter, from a specimen of fluid of hydrocephalus internus which I had collected from the head of a boy who died of that disease under my care; the animal matter having been previously removed by incineration in the manner above described.

1. This saline matter being dissolved in a small quantity of distilled water, and exposed to spontaneous evaporation in a glass capsule, the weather being very warm, and the atmosphere particularly dry, it was found reduced, in the course of two or three days, to the state of a dry crystalline mass, which exhibited a number of large cubic crystals, some of which measured from 8th to 6th part of an inch, mixed with other crystals of the same form, but of much smaller dimensions. In the interstices between these crystals, and on the margin round them, there were observed small white opaque dots, or globules, interspersed with numerous needles, or spicular transparent crystals, perfectly distinct, though of a minute size.

2. The needles, or spicular crystals, were found to be carbonat of soda. The opaque globules proved also to be carbonat of soda. Most of the cubes 
were muriat of soda, but some of the smaller ones were found to be muriat of potash.

3. From another specimen of the same fluid, procured from another dead subject, and treated in a similar manner, the same appearance of cubic crystals interspersed with white specks was obtained; but in this no transparent needles appeared. Here, as in the former specimen, a few small crystals of muriat of potash were found mixed with the muriat of soda, and the white specks, as in the former instance, consisted exclusively of carbonat of soda.

This specimen appeared to contain less muriat of potash than the former, and in both of them the muriat of soda seemed greatly to prevail.

4. The chemical tests which were used for the discovery of potash, and by which this alkali was discriminated from soda, were solutions of tartaric acid, and of oxymuriat of platina*, two re-agents which have the property of forming with potash; in whatever state of combination, salts possessed of but little solubility; so that, if potash be present, a precipitate falls down, and white lines appear on

* Both these tests, provided the solutions be not too dilute, are very distinct in their indications; but I believe that the tartaric acid is capable of discovering more minute quantities of potash than oxymuriat of platina. It is necessary, in the preparation of the latter test, to expel any excess of acid by previous evaporation to dryness. 
scratching the vessel with a pointed instrument*.

5. The carbonat of soda was identified, not only by tests indicative of the absence of potash, but also by its forming rhomboidal, instead of prismatic crystals, when treated with nitric acidt.

6. It may be worth while mentioning, that none of the cubic crystals, when well washed, were found to contain both soda and potash; and that it appeared by comparative trials, that, when mixed solutions of muriat of soda and muriat of potash were caused to crystallize, the cubic crystals obtained consisted either of pure muriat of potash, or pure muriat of soda; the two muriats never being found mixed in the same cube. In some of my first trials, both upon the fluid of spina bifida, and upon that of hydrocephalus, I had been led to conclude that no potash was present, from having

* While correcting this sheet, I learn from my friend Mr. Charles Aikin that the tartaric acid produces, with strong solutions of soda, or of carbonat of soda, precipitates resembling in appearance those obtained from potash or its compounds. But there is this material difference between the two alkalies, that soda forms a supertartrat which is comparatively very soluble; and that the compounds which soda forms with the nitric, muriatic, and acetic acids, are not visibly acted upon by the tartaric acid, whilst all the salts of potash are decomposed by that acid.

+ Nitrat of soda crystallizes in rhombs, whilst nitrat of potash usually appears in the form of bexagonal prisms. 
obtaifed no precipitates by oxymuriat of platina; but this had no doubt arisen from my having operated upon dilute solutions of the entire saline mass, instead of selecting particular crystals*.

7. It was in consequence of Dr. Pearson's assertions (both in conversation and in his valuable papers on expectorated matter), that the uncombined alkali contained in the serum of blood was potash and not soda, contrary to the opinion generally received, that $I$ was induced to direct $m y$ attention to the subject. The statement of Dr. Pearson, that tartaric acid, when added to the saline matter of serum, produces supertartrat of potash, and that therefore potash must be present, was fully confirmed by the experiments just related, in regard to the fluid of hydrocephalus; and the same coinci-

* I cannot refrain from taking this opportunity of acknowledging the great advantages which $I$ have derived from the writings of one of our honorary members, Dr. Wollaston, from his conversation, and occasionally also from his kind personal assistance in this and other similar inquiries. The acuteness with which he discriminates crystalline forms, however minute, the neatness of his chemical manipulations, and the dexterity with which he analyses the smallest quantities of matter, are known only to those who have seen him engaged in experimental researches. The chemistry of microscopic quantities is in a great degree his own. The large, dismal, subterraneous laboratory of old chemists, is now changed for the fire-side of a comfortable study; and a new school is rising under his auspices, and those of two or three other British chemists, which promises to give a most essential impulse to the progress of analytical chemistry. 
dence of results was obtained, as I shall soon notice in detail, when the serum itself was examined. But there is this material difference between Dr. Pearson's conclusions and those which I have drawn from my experiments, that Dr. Pearson has considered the uncombined alkali as consisting exclusively of potash", whilst I believe it to consist exclusively of soda, and consider the small portion of potash present as being combined with muriatic acid.

\$IV. Of the Fluids of Ascites, Hydrothorax, and Hydrops Pericardir.

Whilst I was engaged in the experiment just related, I was favoured by Dr. Okes, of Caius College, Cambridge, with specimens of dropsical fluids from the cavities of the abdomen, of the thorax, and of the pericardium, all of them from the same subjectt. Dr. Okes, who is now attend-

* Since the first edition of this volume, Dr. Pearson has published in vindication of this opinion, several papers in Nicholson's Journal, and in the Philosophical Magazine ; and I have endeavoured to answer his arguments in the same periodical works, partly by new facts, and partly by theoretical reasoning. Professor Berzelius, who has since published a paper on animal fluids, in the third volume of these Transactions, states the alkali to be soda, partly uncombined, and partly united with the lactic acid; and that eminent chemist does not even appear to consider the subject as admitting of doubt or discussion.

+ I have also, in the course of this inquiry, been obligingly. assisted in procuring specimens of fluids, by Mr. Lewis, assistant to Mr. Cooper, and by Messrs. Johnson and Calloway, both pupils to Guy's Hospital. 
ing the medical practice of Guy's Hospital, procured these various fluids from the body of a young man of 17 , who had recently died in the Hospital of an universal dropsy.

These fluids," when first examined, were quite recent and free from smell. They differed much in regard to the quantity of animal matter from either of the preceding fluids; but they bore a close resemblance to each other, both in their external and in their chemical properties, though small differences were observed, as will appear from the following particulars.

A. Fluid from the cavity of the Abdomen.

1. It was of a yellowish colour, inclining to green.

2. Its specific gravity was 1015 .

3. It was alkaline.

4. The mineral acids threw down from it considerable white flaky precipitates.

5. On applying heat, considerable masses of coagulated albumen appeared, though not in sufficient quantities to convert the whole fluid into an uniform solid coagulum.

6. 600 grs. of this fluid being evaporated to dry- 
ness, the residue, when reduced to the half charred state before described, weighed 20,2 grs.

7. In order to acquire more precise information respecting the nature of the animal matter contained in this fluid, a portion of it, equal to about $900 \mathrm{grs}$. was boiled with a little dilute muriatic acid. After the separation of the albumen coagulated by this process, the clear fluid gave no ponderable precipitate with either oxymuriat of mercury, or infusion of galls, though with both it occasioned a slight cloudiness. This clear fluid being evaporated to dryness, yielded 5,1 grs. of salt*, and 2,3 grs. of half charred animal matter (apparently of the mucoextractive kind), independently of the albumen which had been previously separated. During this evaporation a smell arose very much resembling that of urea ; but it should be observed that the fluid under experiment had begun to putrefy.

8. The residual mass (6), after being ignited in a platina crucible, and after being separated from the coaly matter-by re-dissolution, filtration and re-evaporation to dryness in a sand heat, was reduced to 4,8 grs.

9. This saline mass, when caused to crystallize

* The salts were separated from the animal matter, as usual, by incineration. 
slowly, exhibited clusters of crystals, partly cubic, partly octohedral, interspersed with others of a feathery or radiating appearance.

10. The feathery saline matter effervesced briskly with acids, and yielded no permanent precipitate either with tartaric acid or with oxymuriat of platina.

11. The cubic and octohedral crystals, on the contrary, yielded a precipitate with either of the two tests just mentioned.

12. Solutions of barytes appeared to indicate rather a greater portion of sulphuric acid in this than in the former fluids.

13. A portion of the saline mass being treated with dilute nitric acid, and the solution being gently evaporated, a confused crystallization took place, in which rhomboidal crystals were distinctly discoverable.

14. The coaly matter ( 8 ) being treated with dilute muriatic acid, and the filtered solution evaporated to dryness in a sand heat, the saline residue thus obtained weighed 0,3 grs.

15. This residue was found to contain lime, iron, magnesia, phosphoric acid, and a minute quantity of animal matter. 
16. It appears from all these particulars that 1000 grs. of the fluid of ascites* consist of

Water

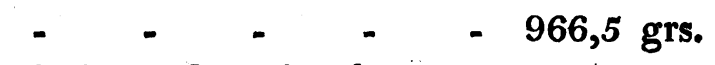

Albumen, desiccated as in the preceding analyses $\quad-\quad-\quad-22,6$

Muco-extractive animal matter $\quad$ - $\quad 2,5$

Muriat of soda, with a little muriat of potash - - - - - 6

Subcarbonat of soda, with some traces

of an alkaline sulphat ${ }_{1} \quad-\quad$ - $\quad 1,9$

Phosphats of lime, iron, and magnesia $\quad 0,5$

1000,0 grs.

which makes a total of $33,5 \mathrm{grs}$. of solid matter in 1000 grs. of the fluid, of which 25,1 grs. consist of animal matter, and 8,4 of saline ingredients.

\section{B. Fluid from the Thorax.}

* The fluid in question was, as I stated before, taken from the cavity of the abdomen. Since the first edition of this volume, I have had the opportunity of examining the fluid of an ovarian dropsy obtained by paracenthesis. It was somewhat viscid, and of a chocolate brown colour. Its specific gravity was 1020,2. It deposited, on standing, a considerable quantity of brown sediment. By heat or acids it was reduced to the state of a thick pulp, but not to that of a firm mass. It contained exactly eight grains of salts in 1000 grs. of the fluid.

† The specimen of fluid used to determine these proportions was from the thorax, as the supply of abdominal fluid happened to be exhausted; but these two fluids resembled one another so closely in their general properties, that there is no reason to suppose they would have been found to differ in regard to the proportion of alkali. 
1. The external appearance of this fluid was nearly the same as that of the former; but on pouring it from one vessel into another, after it had stood for 24 hours, a portion of it appeared heavier than the rest, having somewhat of a pinkish hue, and forming a distinct mass like the white of an egg. By agitation, however, this denser portion appeared to diffuse itself throughout the fluid, and on the fluid being again left at rest for some hours, there subsided a few flakes of a selfcoagulable substance, which all albuminous fluids are apt to deposit.

2. The specific gravity of that fluid was 1012,1.

3. On being evaporated and desiccated in the same way as the former, it yielded only $26,6 \mathrm{grs}$. of solid matter in 1000 of the fluid; $18,8 \mathrm{grs}$. of which consisted of animal matter, and 7,8 grs. of saline ingredients, namely, 6 grs. of muriats, and $1,8 \mathrm{grs}$. of carbonated alkali.

In all other respects, so far as it was examined, this fluid appeared perfectly similar to the former.

\section{Fluid from the Pericardium.}

1. Its specific gravity was 1014,3 .

2. It yielded $\mathbf{3 9}$ grs. of solid matter in 1000 of the fluid; namely, 25,5 grs. of animal matter, and 7,5 grs. of salts. 
In its external appearance, and in every other respect, no difference was observed between this and the two preceding fluids.

\section{$\S \mathrm{V}$. Of the Serum of the Blood.}

The serum of blood has so often been the subject of chemical examination, that I should have thought it superfluous to have turned my attention to it, had I not been desirous of obtaining, respecting this fundamental fluid, some general results more immediately comparable with those which I have just related respecting the morbid serous secretions. We are indebted to Dr. Bostock for an accurate examination of the animal matter contained in the blood, and for the curious discovery of the absence of gelatine in the serum*, which . my own trials have fully confirmed. I shall therefore chiefly direct my attention to the saline contents of the serum, such as obtained by incineration; previous to which, however, I shall shortly relate my attempts to ascertain the specific gravity of serum, and the proportions of albumen and extractive matter which it contains.

* See Medico-Chirurgical Transact. vol.I. and II. If any coincidence should be observed in my researches on serum, with those of Dr. Bostock, published in this volume of the Society's Transactions; or if either of the two essays should appear to have derived any assistance from the other, I think it right to mention that I have had, through the kindness of Dr. Bostock, and from my situation as one of the secretaries, the advantage of a free access to Dr. Bostock's manuscript, whilst I was finishing my own paper; so that I am alone responsible for any repetition or redundance which may have occurred in the course of our inquiries. 
A. I found the specific gravity of serum to be subject to remarkable variations. The first specimen which I examined was procured from a healthy person who had been bled for a slight local injury. Its specific gravity was 1024,5 . The next specimen was obtained from a stout young woman labouring under acute rheumatism, attended with pulmonic affection. This blood was not buffy, though the symptoms were indicative of inflammation; the specific gravity of the serum was 1032,5 . A third specimen was obtained from the same patient two days afterwards : the blood had this time a thick buffy coat on its surface, and the specific gravity of the serum was 1 (29,8. A fourth specimen procured from a patient labouring under a fe.brile affection was exactly of the same specific gravity as the last, namely, 1029, 8 ; this blood had but a very slight buffy coat, and the serum was somewhat turbid, like whey. In a fifth specimen of serum, from a mass of blood which did not exhibit the smallest degree of inflammation, the specific gravity proved to be 1030,9 .

The mean specific gravity of serum, therefore, resulting from these five observations would be 1029,5 , the two extreme points being 1024,5 and 1032,5 .

B. Proportions of coagulable and uncoagulable animal matter in Serum. I attempted to ascertain these proportions in the following manner: 
1. 500 grs. of recent serum of the specific gravity of 1029,8 being exposed to the heat of a lamp, soon passed to the state of a white coagulated mass, apparently firmer and containing a smaller proportion of uncoagulable serosity than any of the preceding fluids. This coagulum being gradually desiccated, and ultimately brought to the half charred state often described, weighed 50 grs.

2. 500 grs. of serum, from the same specimen, were boiled with a few drops of muriatic acid, so as to coagulate the albumen completely; water was then added to the coagulated mass in order to wash off the whole of the serosity. This was effected, not by filtering, for the liquor passed through the filter milky, but by slow spontaneous separation, and decantation. The clear fluid was no longer acted upon by oxymuriat of mercury, and in evaporating gave no smell of urea. The residue, when brought to the state of a half charred brittle mass, weighed 3,6 grs. from which 1,6 grs. of salt were afterwards obtained by incineration. The proportion of muco-extractive matter, therefore, thus desiccated, would only be 2 grs., and that of albumen about 44 grs. in 500 grs. of serum.

C. Saline contents of the Serum of the Blood.

1. 1000 grs. of recent serum, of the specific gravity of 1024,5, being incinerated in the usual 
mode, the soluble salts, separated by water, and desiccated to incipient ignition, weighed 7,7 grs. This saline matter was alkaline, and amongst the confused crystalline forms which it exhibited, distinct octohedral crystals were observed, though no smell of urea had been observed during the evaporation. This mass soon deliquesced on being exposed to the air.

2. The coaly mass being treated with dilute muriatic acid, and the clear acid solution evaporated to dryness in a sand heat*, the saline residue weighed 1,5 grs., which, with the 7,7 grs. obtained by the first operation, makes 9,2 grs. of salts in 1000 grs. of serumt.

3. About two-thirds of this last residue appeared to consist of cubic crystals of muriat of soda, which had remained attached to the insoluble salts after incineration, and had escaped the action of the

* By a sand heat I have meant, throughout this paper, the greatest heat which can be communicated to a small saucer containing a little sand, by a lamp of moderate power : the degree of dryness thus obtained is telerably uniform, and very nearly equals, in the case of fixed salts, that which is obtained by heating the naked vessel to incipient redness.

+ In one instance in which the specific gravity of the serum was 1032,5 , the whole quantity of saline matter was only $6 \mathrm{grs}$; so that if any conjecture may be hazarded upon a single result of this kind, the natural inference would be that the denser serum is, the less saline matter it contains. 
watery solvent. The remaining third proved to consist of iron, lime and magnesia, combined with phosphoric acid. All these substances were distinctly detected by their respective agents. The presence of magnesia, in particular, was discovered in this mass, by dissolving a portion of it in muriatic acid, precipitating the lime by oxalat of ammonia, and adding to the filtered solution, neutral carbonat of ammonia. On scratching the vessel, the appearance of white lines, which characterizes the formation of an ammoniaco-magnesian phosphat, was distinctly perceived.

4. The saline mass soluble in water (1), consisted chiefly, as in all the preceding fluids, of muriat of soda, with some uncombined alkali. It also contained a distinct trace of the sulphuric and phosphoric acids, which shewed the presence of minute portions of an alkaline sulphat and phosphat. Neither lime nor iron could be detected in this mass.

D. Nature and proportion of the Alkali contained in Serum. The next, and indeed the principal objects which I had in view in examining the serum, were to ascertain the nature of the uncombined alkali contained in it, and the proportion of this alkali.

1. From a concentrated solution of the saline mass (C. 4.), oxymuriat of platina did not produce a precipitate sufficiently distinct to be conclusive VOL. II. 
as to the presence of potash ; but by means of tartaric acid, a distinct, though not abundant, precipitate' was produced.

2. A portion of this saline mass being dissolved in nitric acid, and the solution evaporated to dryness, a deliquescent crystalline mixture was obtained, in which the thomboidal form was conspicuous.

3. Another portion of the same mass being dissolved in acetic acid, evaporated to dryness, and afterwards treated with alcohol and evaporated again, the alcoholic residue, contrary to my expectation, exhibited traces of potash, both by means of the tartaric acid and of oxymuriat of platina *. But the same residue treated with nitric acid was almost entirely resolved into rhomboidal crystals, amongst which I was unable to detect any distinct prisms; whilst on the contrary, potash was easily discovered in the residue insoluble in alcohol, which residue had now lost its deliquescent quality.

4. It appears, therefore, that the uncombined alkali contained in the serum, is (as in the case of

* This result may appear scarcely compatible with that which follows; but I have reason to suspect, from some comparative trials, that this appearance of potash in the alcoholic solution was owing to a few particles of muriat of potash taken up by the spirituous menstruum. 
all the other fluids), soda and not potash, and that the small portion of potash contained in the serum of blood exists there in the state of muriat *.

5. The proportion of the uncombined soda contained in serum was ascertained by a method which appears to admit of great accuracy. To $10 \mathrm{grs}$. of ignited saline matter from serum of blood, a known bulk of very dilute muriatic was added, which was more than sufficient to neutralize the solution, and a piece of marble of known weight was thrown into this solution to neutralize the redundant acid. On the other hand, a similar measure of acid being poured upon another piece of marble, and the diminution of weight of both pieces of marble compared, that which had been treated with muriatic acid alone was found to have lost 1,9 grs. more than the other; and as $1,9 \mathrm{grs}$. of carbonat of lime correspond to $1,05 \mathrm{grs}$. of ignited muriatic acid, and to 1,23 grs. of soda $t$, it was inferred that $10 \mathrm{grs}$. of the saline mass in question contained 1,23 grs. of pure caustic soda, which is equivalent to about $1,8 \mathrm{grs}$. of the subcar-

* Or perhaps also of sulphat, as will be discussed bereafter.

+ The data upon which these statements are grounded, are detailed in my Analysis of the Dead Sea, in which it is shewn that 100 grs. of pure marble contain 56,1 grs. of lime; $100 \mathrm{grs}$. of muriat of lime contain $50,77 \mathrm{grs}$. of the earth, to $49,23 \mathrm{grs}$. of the acid; and 1000 grs. of muriat of soda contain 46 parts of acid, to 54 of alkali. (Philos. Transact. 1807.) 
bonat*, a proportion which does not materially differ from those obtained by different methods in the analysis of the dropsical fluids.

E. Nature and proportion of the Alkaline Sulphat contained in Serum t.

1. $10 \mathrm{grs}$. of the ignited saline matter of serum being dissolved in water, and neutralized by muriatic acid, muriat of barytes precipitated from this solution 0,5 grs. of ignited sulphat of barytes.

2. It now becami a question to decide whether the alkaline sulphat contained in the serum, as well as in all the other animal fluids, was a sulphat of potash or a sulphat of soda. The superior attraction of sulphuric acid for potash rendered the first of these suppositions most probable; and it was farther confirmed, by observing, that by mixing together solutions of muriat of potash and sulphat of soda, and evaporating the mixture, sulphat of potash was obtained in dodecahedral crystals.

* Subcarbonat of soda consists, according to Mr. Kirwan, of 2 parts of acid to 3 of alkali. The present result, however, does not admit of an accurate comparison with those obtained in the analyses of the dropsical fluids; since by the method which was then employed, the soda was neither in a caustic state, nor in a state of definite combination.

† According to Professor Berzelius, sulphuric acid does not originally exist in the animal fluids, but is formed by the process of incineration. 
3. In order to form an estimate of the quantity of sulphat of potash corresponding to this precipitate, 20 grs. of ignited sulphat of potash, prepared on purpose from pure ingredients, were dissolved in water, and decomposed by muriat of barytes.The ignited precipitate weighed $26,4 \mathrm{grs} .{ }^{*}$ Therefore, the quantity of sulphat of potash contained in $10 \mathrm{grs}$. of the saline matter of serum, appears to be about 0,4 grs.

F. Recapitulation. On reviewing and connecting together the foregoing statements on the subject of serum, we may conclude that 1000 grs. of this fluid consist of the following ingredients : viz.

\section{Water - - - - - 900 grs.}

Albumen, reduced by a sand heat to the state of a dry brittle mass, as in the former analyses - $\quad-86,8$

Muco-extractive matter dried in the same way $\quad-\quad-\quad-\quad-4,0$

Muriat of soda, with a little muriat of potash - - $\quad-\quad-\quad-6,6$

Subcarbonat of soda $\quad-\quad-\quad-\quad 1,65$

Sulphat of potash _ - - : 0,35

Phosphats of lime, iron, and magnesia $\quad 0,60$

1000,00 grs.

* So that 100 grs. of sulphat of potash would give 132 grs. of ignited sulphat of barytes. 
which makes a mass of 100 grs. of solid matter in 1000 of serum, $90,8 \mathrm{grs}$. of which consist of animal matter, and 9,2 grs. of saline ingredients.

$\S$ VI. Summary Account of some other Dropsical Fluids.

A. Fluid of Hydrocele. A specimen of this fluid, with. which I was favoured by Mr. Cooper, had the following properties :

1. It was perfectly clear and transparent, resembling in appearance pale sherry wine. Its specific gravity was 1024,3 . It had no smell, and deposited no sediment whatever on standing for a few days. After a week, however, it became slightly turbid, and on removing the cork it exhaled a strong smell, resembling that of phosphorated hydrogen.

2. The dilute mineral acids produced only a slight turbidness; but in their concentrated state, they produced abundant white precipitates. Oxymuriate of mercury and alcohol threw down copious white precipitates. Infusion and tincture of galls both produced considerable brownish precipitates.

3. Heat and concentration occasioned first an opacity and then a thickening of the fluid mass, which, by stirring, assumed the form of a white semi-solid pulp, like thick paste; and by continu- 
ing the heat, the mass passed to the state of a firm coagulum.

4. Some of the serosity, or uncoagulable part of the fluid, being separated, and farther concentrated, did not gelatinize on cooling; yet it still became turbid, though in a much slighter degree than the original fluid, on adding infusion of galts. This serosity being boiled with oxymuriat of mercury, according to Dr. Bostock's method, in order to separate the last portions of albumen upon which I suspected the effect of galls to depend, a milkiness appeared, which could not be entirely removed by filtering; but on adding infusion of galls to the filtered fluid, no further effect could be discovered.

5. 500 grs. of this fluid being evaporated to dryness, and incinerated in the usual way, yielded a saline mass, which, dried at a sand heat, weighed 4,1 grs.

6. $200 \mathrm{grs}$. of the same fluid being evaporated and reduced to the half charred state often described, weighed 16 grs., which makes a proportion of $80 \mathrm{grs}$. of saline matter in $1000 \mathrm{grs}$. of the fluid, 71,5 grs. of which consisted of animal matter, and $8,5 \mathrm{grs}$. of saline ingredients ${ }^{*}$.

B. Fluid of a Hydatid.' A quantity of this

* Allowing 0,3 grs. for the saline matter contained in the coaly mass. 
fluid was procured from a hydatid attached to the kidney of a woman whose body was opened by Mr. Cooper and myself at Guy's Hospital. It was clear and transparent, though of a yellowish colour. No coagulum or turbidness appeared on adding dilute sulphuric or muriatic acid ; but concentrated muriatic acid produced a milkiness. Infusion of galls and oxymuriate of mercury occasioned precipitates. Heat did not produce any coagulation, except after very considerable concentration. The specific gravity of this fluid was not ascertained ; but 1000 grs. of it being evaporated to dryness at a temperature not exceeding $180^{\circ}$, the residue weighed 36 grs. *, and yielded by incineration a saline mass weighing $8,7 \mathrm{grs}$. This saline mass contained the usual ingredients, namely, muriat of soda crystallized chiefly in octohedrons, phosphat of iron and of lime, and a small portion of sulphuric acid.

Upon the whole this fluid, which was examined long since, and with much less minuteness than the others, appears to resemble much in its chemical composition that of hydrocephalus and spina bifida, only containing a larger proportion of animal matter, which was chiefly of the muco-extractive kind, since it did not either

- This residue having, by an accidental oversight, been desiccated at a lower temperature than the other fluids, the result is not immediately comparable to those obtained in the other anylyses. 
coagulate by heat, or gelatinize by cold and concentration.

\section{Fluid from a Tumor in the Thyroid Gland.} The fluid in question was discharged after death, from a cyst formed in each lobe of the thyroid gland of a middle-aged woman who died at the hospital. The whole of the fluid contained in these tumors amounted to between 2 and 3 ounces. It was of a reddish brown colour, not unlike that of a decoction of bark, this colour being evidently not occasioned by any accidental admixture of blood. The fluid was unctuous to the touch, and innumerable small micaceous particles were $\cdot$ seen floating in it*. Its specific gravity was 1031,8. Paper stained with violets indicated the presence of uncombined alkali. Heat converted it into a firm coagulum of a greyish white colour. It was also readily coagulated by acids and by oxymuriat of mercury, and yielded a copious precipitate on adding an infusion of galls.' On adding caustic potash, the fluid continued clear, and no smell of

* A portion of this fluid having been allowed to stand till it was beginning to putrefy, a sediment was observed to subside from it. The supernatant liquor being decanted off, the sediment was spread upon a card, where it soon dried, leaving a glossy shining film upon the card. This substance being scraped off and exposed to the heat of a candle, burnt with a bright flame, and apparently withont undergoing previous fusion. Another portion being treated with alcohol, appeared to dissolve partly in it, and reappeared on adding water, or evaporating the alcohol. 
ammonia arose from it. On separating the incoagulable serosity by ablution with water, and evaporating till nothing but a dry extract remained in the vessel, no gelatinization took place' by cooling at any period of the operation, but there arose a strong offensive smell, resembling that of urea *.

On heating the coagulum to redness, the mass swelled, bubbled up, and finally burnt as usual, emitting a smell resembling that of burning horn, and leaving a coaly residue.

The whole of the salts contained either in the serosity or in the albuminious part, being in the usual way separated by incineration, amounted only to $5 \mathrm{grs}$. in 1000 of the fluid, a smaller proportion than that yielded by any of the other fluids $t$. But in their nature, these salts appeared to be in every respect similar to those of the other fluids before described.

D. Fluid from a Tumor in the Chest. The circumstances attending this tumor were singular. A female patient in Guy's Hospital, under the care of Dr. Curry, was labouring under an op-

* The fluid was, at this time, beginning to putrefy.

+ The separation of the salts was not, in this flujd, of which I had but a very scanty supply, conducted with the same accuracy as in the other fluids. For this reason $I$ have not inserted this result in the table at the end of this paper. 
pression in her breathing, and other symptoms resembling those of Hydrothorax, though not in any urgent degree. Upon examination, a distinct and considerable protuberance was observed on the right side of the chest, in consequence of which the ribs themselves were thought to protrude. A puncture being made with $a$ lancet in the pectoral muscles, where the tumor appeared the most prominent, about a pint of fluid, perfectly clear and colourless, was discharged, and the woman rapidly recovered.

The specific gravity of this fluid was 1008,5, and upon minute examination it was found to resemble closely in its properties the fluid of $\mathrm{Hy}$ datids or that of Hydrocephalus; and it differed so much from that of Hydrothorax in regard to the quantity of animal matter and the proportion of albumen, as well as to its external appearance, as to render it highly probable that the disease depended upon some peculiar èncysted effusion on the surface, and not, as was suspected, within the cavity of the chest.

With respect to the saline contents, they amounted to $9 \mathrm{grs}$. in 1000 of the fluid, and appeared to be perfectly similar to those of the other fluids before examined.

$\S$ VII. General View of all the preceding Results.

As there are but few readers to whom chemical details are interesting, and as most of them 
may be glad to obtain some general notions of the results, without wading through a mass of experimental evidence, it may be proper to retrace, in a few words, the leading points of this inquiry.

It appears in the first place, that the prevailing animal substance,' not only in serum, but in all the morbid fluids which have been examined in this essay, is albumen, or coagulable matter; which substance, however, these fluids contain in very different proportions.

In all of them, also, another kind of animal substance which may be called muco-extractive matter, (from its being incoagulable, and from its being soluble in water or other menstrua,) is uniformly discoverable.

Gelatine, it would appear, is not discoverable in any of these fluids *; a singular circumstance, from which it seems natural to infer that the formation of gelatine is the result of a specific secretion.

In some of these fluids, namely, those of ascites, hydrothorax, hydrops pericardii, hydrocele, and

* Since the publication of the first edition of this volume, I have also examined the fluid discharged by blistered surfaces, in which I have not been able to detect the least vestige of gelatine. The specific gravity of this fluid, and its proportion of saline matter, will be found in the table at the end of this paper. 
that which is sometimes effused in the thyroid gland, the albuminous matter is so considerable as to render them coagulable; that is, convertible into an uniform semi-solid mass, by the agency of acids, or by a temperature of 165 degrees. In others, on the contrary, namely, in the fluid of spina bifida, of hydrocephalus, and of hydatids, the quantity of albuminous matter is so small, as scarcely to be rendered visible by heat or acids.

The specific gravity of these fluids is likewise remarkably various. That of the fluid of hydrocephalus, for instance, is under 1007 ; whilst that of serum sometimes exceeds 1032. Different specimens of the same fluids are also found to vary in their specific gravities. This is especially conspicuous with regard to the serum of blood, some specimens of which do not exceed 1024, whilst others rise to 1032,5. Even in the same individual, and during the continuance of the same disorder, differences of specific gravity are observable in serum obtained at different periods. Thus, also, it is found that dropsical fluids from different cavities of the body, collected after death from the same individual, differ materially in regard to their specific gravities.

Upon farther examination it is found that these differences affect principally, and perhaps exclusively, the animal matter of these fluids; the saline matter contained in them not being subject to similar variations. 
These saline contents, on the contrary, are found to be remarkably uniform in the different fluids, both as to their proportions and their chemical nature; and the same uniformity prevails whatever differences there may exist in these fluids in regard to the animal matter they contain. Thus, serum of blood, and the fluid of hydrocephalus, yield the same saline substances, and very nearly in similar proportions, although the one contains about eighty times as much animal matter as the other *. The proportion of saline matter yielded by the various animal fluids concerned in this inquiry, may be generally stated to be between 8 and 9 grs. in 1000 grs. of fluid.

The particular saline ingredients contained in all these fluids appear to be, muriat of soda, muriat of potash, sulphat of potash, soda, and phosphats of lime, iron and magnesia. And a mass of $100 \mathrm{grs}$. of these salts appears to consist of about 72 grs. of muriat of soda, mixed with a little muriat of potash; between 18 and $20 \mathrm{grs}$. of soda, brought to the state of subcarbonat; and a mixture of 8 or $10 \mathrm{grs}$. of sulphat of potash, phosphat of lime, phosphat of iron, and phosphat of magnesia. Potash, therefore, as Dr. Pearson has stated, is present in the animal fluids; but I believe I have satisfactorily shewn, that it exists in the state of muriat, or sulphat; soda being the only alkali discoverable in an uncombined state.

- A table, exhibiting the general results of all the above analyses, will be found at the end of this paper. 
The caustic alkali contained in these fluids appears to be combined with their animal matter, the properties of which it modifies in a manner which is not yet well understood*: and it would seem that the proportion of uncombined alkali to that of the other salts is greater, as the specific gravity of the fluid is more considerable.

These circumstances, and in particular the remarkable variations which are observed in the proportions of animal matter contained in the blood of different persons, or of the same person at different periods, derive considerable interest from their probable connection with health and disease, and from the new pathological views which a full investigation of the subject might suggest.

- Mr. Brande, in his "Observations on Albumen, \&c." published in the Philosoph. Transact. for 1809 , has made an ingenious attempt to explain the agency of alkali in albuminous fluids; and Professor Berzeljus, in his interesting paper on Animal Fluids, which was published in the third volume of the Society's Transactions, previous to the reprinting of this volume, has considered the alkali of the animal fluids as neutralized, almost entirely, by the lactic acid. From my method of operating, I could not be led to suspect this mode of combination, since in separating the salts from the animal matter by incineration, any destructible acid would necessarily escape detection. 
384 ACCOUNT OF VARIOUS DROPSICAL FLUIDS.

\section{TABLE}

Shewing the proportions of saline and animal matter in various dropsical or serous fluids, and in the serum of blood.

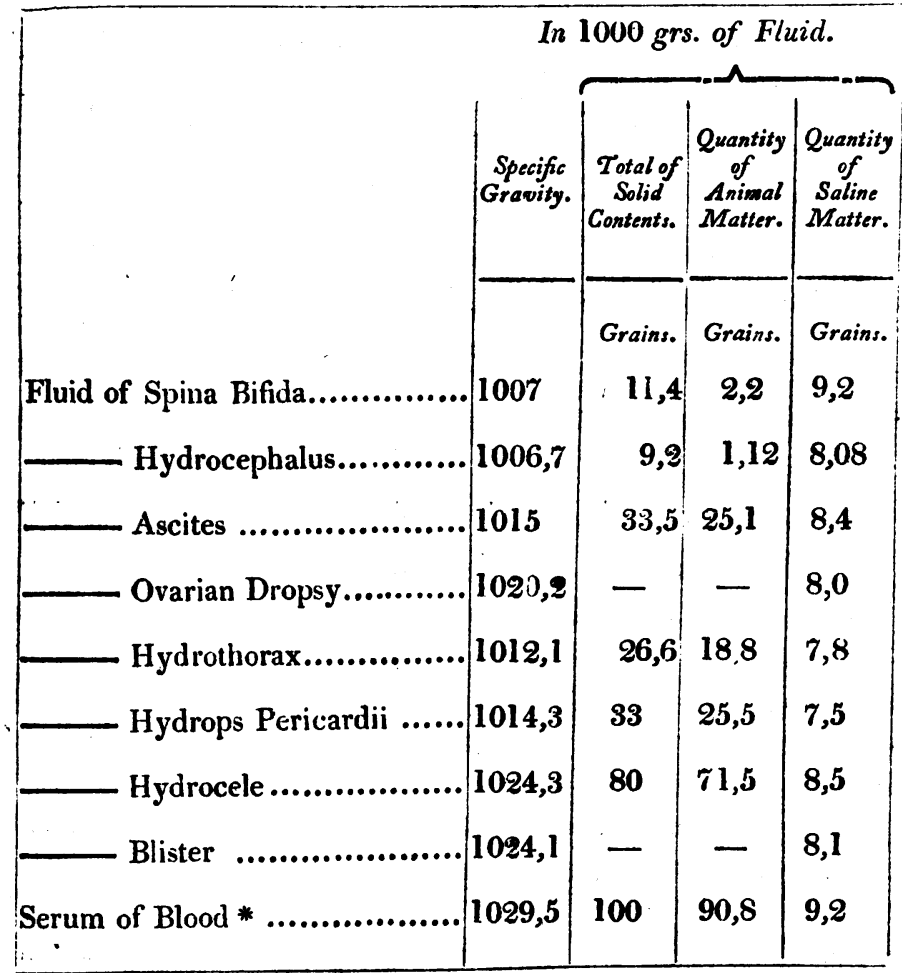

* The specific gravity here stated is an average. That of the particular specimens under examination was 1024,5 , as to the saline substances ; and 1029,8 , as to the animal matter.

P. S. I have, since the first publication of this paper, taken the specific gravity of ten specimens of human serum, which yielded a mean specific gravity of 1029,0 . The heaviesh specimen was from a diabetic patient, and weighed 1035,4; the lightest, which was taken from an emaciated consumptive person, weighed 1027,1 . 